Journal of Animal and Veterinary Advances 11 (8): 1258-1264, 2012

ISSN: $1680-5593$

(C) Medwell Journals, 2012

\title{
Liver Cells of GFP Transgenic Male Mice Transplantation to Reconstruct Hematopoietic Function in Female Mice
}

\author{
Guang-Ping Ruan, Jin-Xiang Wang, Rong-Qing Pang, \\ Xiang Yao, Yong-Li Deng, Qiang Wang, Li-Hua Ma and Xing-Hua Pan \\ Kunming General Hospital of PLA, The Research Center of Stem Cell, \\ Tissue and Organ Engineering, 650032 Kunming, China
}

\begin{abstract}
The liver cells of fluorescent male mice were transfused to half-lethally irradiated C57BL female mice. Whether liver cells had hematopoietic reconstruction function was investigated. Researchers used liver cells of fluorescent male mice to transfuse back to half-lethally irradiated C57BL females mice. At 18, 39, 53 days after transplantation, mice were cut tails to collect blood and with CD4-PE and CD8-PE labeled and then bloods were hemolyzed by ammonium chloride. Then, the cells were detected by flow cytometry. About 87 days after transplantation, mice were killed to collect bone marrow and detected $\mathrm{Y}$ chromosome positive percentage by fluorescence in situ hybridization. GFP positive cells were detected in peripheral bloods, spleen cells, liver cells and bone marrow cells of three sacrificed mice. The female mice infused with liver cells were detected GFPpositive cells in peripheral blood and some GFP-positive cells converted into CD4+ or CD8+T cells. The percentage of GFP-positive cells in blood was positive correlated to the proportion of $\mathrm{Y}$ chromosome in bone marrow detected by fluorescence in situ hybridization analysis. GFP positive cells can be detected in peripheral bloods, spleen cells, liver cells and bone marrow cells of three sacrificed mice. The percentages of GFP-positive cells detected in the spleen cells were higher than other cells with statistical significance $(p=0.003)$. The adult mice liver cells contained a certain amount of hematopoietic stem cells. These cells survived in female mice and distributed in various organs and could reconstruct hematopoietic function.
\end{abstract}

Key words: GFP transgenic mice, liver cells, fluorescence in situ hybridization, Y chromosome, hematopoietic reconstitution, China

\section{INTRODUCTION}

Fluorescent mouse is GFP transgenic mouse. All the cells of bodies are with fluorescence. After the cells from fluorescent male mice were transplanted to female mice if the transplanted cells survived, it can be proved by two ways. One way is to detect the percentage of GFPpositive cells in peripheral blood and various organs. The other way is to detect the percentage of Y chromosomepositive cells in the bone marrow cells. In this experiment, researchers used liver cells of adult fluorescent male mice transfused back to half-lethally irradiated female mice to study whether the adult mice liver cells can reconstruct the hematopoietic function. It is reported fetal liver cells contain hematopoietic stem cells while whether adult mice liver cells contain hematopoietic stem cells or these cells can reconstruct the hematopoietic function are still unclear. It is reported in the literature the muscle cells can rebuild of hematopoietic function in mice (Pang, 2000). And whether the adult mice liver cells can reconstruct hematopoietic function need to be studied.
According to the literature, liver cell transplantation has the potential to treat acute and chronic liver failure and metabolic defects (Lysy et al., 2008; Meyburg et al., $2009 \mathrm{a}, \mathrm{b}$ ). It is an alternative method to treat patients with liver failure awaiting liver transplantation. Usually liver cell transplantation is by injection into the spleen pulp, splenic artery, splenic vein or portal vein (Najimi and Sokal, 2004; Nyamath et al., 2007). Researchers transplanted liver cells by tail vein to female mice and observed survival and distribution of liver cell in vivo in female mice. It will afford some instruction significance for liver cells transplantation to treat diseases.

We prepared adult fluorescent mice liver cell suspension, transfused back to the half-lethally irradiated C57BL female mice. About 18, 39, 53 days after transplantation, GFP positive cells can be detected in peripheral blood and some cells converted into CD4+ or CD8+ $\mathrm{T}$ cells which indicating that these cells have roles to reconstruct hematopoietic function. At 87 days after transplantation, three transplanted mice were sacrificed and their peripheral blood, liver, spleen and bone marrow

Corresponding Author: Xing-Hua Pan, Kunming General Hospital of PLA, The Research Center of Stem Cell, 
cells were collected to detect GFP-positive cells. GFPpositive cells rates in the spleen were higher than in the bone marrow, liver and peripheral blood. And the results of the comparison was statistically significant $(\mathrm{p}=0.003)$. $\mathrm{Y}$ chromosome-positive cells can be detected in bone marrow in female mice by fluorescence in situ hybridization method and the positive rate was proportional to the percentage of GFP-positive cells in peripheral blood. It further proved that the adult mice liver cells contained hematopoietic stem cells can rebuild female C57BL mice hematopoietic function. About 87 days after transplantation, most of the GFP-positive cells concentrated in the spleen to play the role of hematopoietic reconstitution. Whether liver cells contained hematopoietic stem cells or liver cells transdifferentiated into hematopoietic stem cells in vivo after transplantation need further detailed study.

\section{MATERIALS AND METHODS}

GFP transgenic male mice were from Professor Tinghua Wang of Kunming Medical University as gifts. C57BL female mice were purchased from Experimental Animal Center of Third Military Medical University. Experimental protocols were approved by the Experimental Animal Ethics Committee of Kunming General Hospital.

Preparation of erythrocyte lysis buffer: $\mathrm{NH}_{4} \mathrm{Cl} 8 \mathrm{~g}$, $\mathrm{NaHCO}_{3} 0.84 \mathrm{~g}, \mathrm{Na}_{2}$ EDTA $0.37 \mathrm{~g}$ distilled water added to $1000 \mathrm{~mL}$.

C57BL female mice irradiation: About 10 C57BL female mice in toal were irradiated by the linear accelerator with

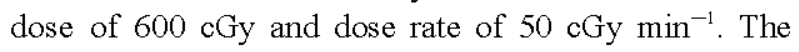
distance to box top was $98.5 \mathrm{~cm}$ with $100 \mathrm{~cm}$ away from the mice center. About 7 mice were transfused liver cell. About 3 mice were transfused saline.

Preparation of liver cells suspension of fluorescent male mice: Sterile liver was taken and soaked in double antibiotics and put on the 100 line screening with the syringe needle core crushing when washed with basal medium. The cells were collected by filtration screen and hemolyzed with erythrocyte lysis buffer. After cells were counted, each mouse was injected with $5 \times 10^{6}$ liver cells via the tail vein.

The percentages of GFP positive cells in peripheral blood were detected: Mice tails were cut and the blood was dropped into the EP tube with $100 \mu \mathrm{L}$ anticoagulants, mixed evenly, centrifuged and anticoagulants were aspirated. Red blood cells were hemolyzed with ammonium chloride solution. The percentages of GFPpositive cells were detected by flow cytometry.

GFP and CD4-PE (purchased from biolegend) or CD8-PE (purchased from biolegend) double positive cells were detected in peripheral blood: Mice tails were cut and the blood dropped into the EP tube with $100 \mathrm{uL}$ anticoagulants, mixed evenly, centrifuged and anticoagulants were aspirated. Blood was divided into two tubes and one tube was added with $1.5 \mu \mathrm{L}$ CD4-PE while another tube was added with $1.5 \mu \mathrm{L}$ CD8-PE. Cells were incubated at room temperature for $30 \mathrm{~min}$. Then, red blood cells were hemolyzed with ammonium chloride for flow cytometry analysis of GFP and PE double positive cells.

Detection of Y-chromosome-positive rate in bone marrow cells: Biotin-labelled mouse Y chromosome specific probe (50-100 ng) was made up to $12 \mathrm{~mL}$ with hybridization buffer ( $50 \%$ deionized formamide, $10 \%$ dextran sulphate, $2 \times \mathrm{SSC}, 0.5 \mathrm{~mol} \mathrm{~L}^{-1}$ phosphate buffer, $\mathrm{pH} 7.3$ ). The probe was denatured at $65^{\circ} \mathrm{C}$ for $10 \mathrm{~min}$ and preannealed by incubation at $37^{\circ} \mathrm{C}$ for $30-60 \mathrm{~min}$. Slides were denatured by incubation in $70 \%$ formamide $/ 2 \times \mathrm{SSC}$ solution at $65^{\circ} \mathrm{C}$ for 1-2 min, quenched in ice-cold $70 \%$ ethanol and dehydrated through a 70,90 and $100 \%$ ethanol series. The preannealed probe was applied onto slides and allowed to hybridize overnight at $37^{\circ} \mathrm{C}$. Post-hybridization washes were two $5 \mathrm{~min}$ incubations in $50 \%$ formamide $/ 2 \times \mathrm{SSC}$ at $45^{\circ} \mathrm{C}$ followed by two $5 \mathrm{~min}$ incubations in $2 \times \mathrm{SSC}$ at $45^{\circ} \mathrm{C}$. Biotin-labelled probe was visualized using one layer of Cy3-avidin (1:1000 Amersham). After detection, slides were mounted in Vectashield mounting medium with DAPI (4'6-diamidino-2-phenylindole). Images were captured using the CytoVision system (Applied Imaging) with a CCD camera mounted on a Zeiss Axioplan 2 microscope.

About 8.87 days after transplantation, three of the female mice with liver cells transfusion were collected blood from tail vein and then the mice were sacrificed, the liver and spleen were put on the 100 line screening with the syringe needle core crushing when washed with saline. Filtrations of the cells were collected and hemolyzed and then detected the percentage of GFPpositive cells by flow cytometry and femurs were washed and collected bone marrow cells and hemolyzed and then detected the percentage of GFP-positive cells by flow cytometry.

\section{RESULTS AND DISCUSSION}

At different days after transplantation, to detect the percentage of GFP-positive cells in peripheral blood: at 18 , 
39 and 53 days after transplantation, researchers had cut mice tails to collect peripheral blood, hemolyzed with ammonium chloride, the percentage of GFP-positive cells were detected. About 18 days after transplantation, the percentages of GFP-positive cells of three mice were averaged $26.55 \%$. About 39 days after transplantation, the percentages of GFP-positive cells of 3 mice were averaged $6.5 \%$. About 53 days after transplantation, the percentages of GFP-positive cells of 3 mice were averaged $4.95 \%$. While the other three mice with the saline transfusion, the percentages of GFP-positive cells were $0 \%$ in peripheral blood (Fig. 1).

About 18 days after liver cells transplantation, peripheral blood GFP and CD4-PE double-positive percentage was of $1.5 \%$, GFP and CD8-PE double-positive percentage was of $0.6 \%$. Without transfusion, double positive cells were 0.2 and $0 \%$ (Fig. 2). It showed $4.7 \%$ of the survival of transplanted liver cells in vivo was converted into $\mathrm{CD} 4+\mathrm{T}$ cells, $2.8 \%$ was converted into CD8+T cells.

About 53 days after liver cells transplantation, peripheral blood GFP and CD4-PE double positive percentage was of $0.2 \%$, GFP and CD8-PE double-positive percentage was of $0.2 \%$. The mice without transfusion double positive cells were 0 and $0.1 \%$ (Fig. 3). It showed the survival of liver cells in vivo had $3.8 \%$ converted into CD $4+\mathrm{T}$ cells, $4.1 \%$ converted into CD $8+\mathrm{T}$ cells. About 39 days after transplantation had similar results to 53 days; so it is no longer affording figures.

About 87 days after transplantation, GFP-positive cells were detected in peripheral blood, liver, spleen and bone marrow of three mice. The results were as follows (Fig. 4 and 5). About 87 days after transplantation, the results showed that in the spleen cells had the highest percentage of GFP-positive cells, the results of pairwise comparison showed that the GFP cells positive rate in spleen cells was statistically significant higher than in other cells $(p=0.003)$ and the percentage of GFP-positive cells in peripheral blood, liver and bone marrow compared no significant difference ( $\mathrm{p}>0.05)$.

About 87 days after transplantation, fluorescence in situ hybridization results showed that female mice had $2 \% \mathrm{Y}$ chromosome positive metaphase cleavage image in bone marrow. Analysis of 100 chromosomes metaphase cleavage image, $\mathrm{Y}$ chromosome was detected in 2 metaphase cleavage image (Fig. 6) and $1.7 \%$ of GFPpositive cells in peripheral blood were positively correlated to the $\mathrm{Y}$ chromosome-positive rate in bone marrow.

The results showed that after the liver cells transplantation, GFP-positive cells can be detected in peripheral blood. Whether because the liver cells self

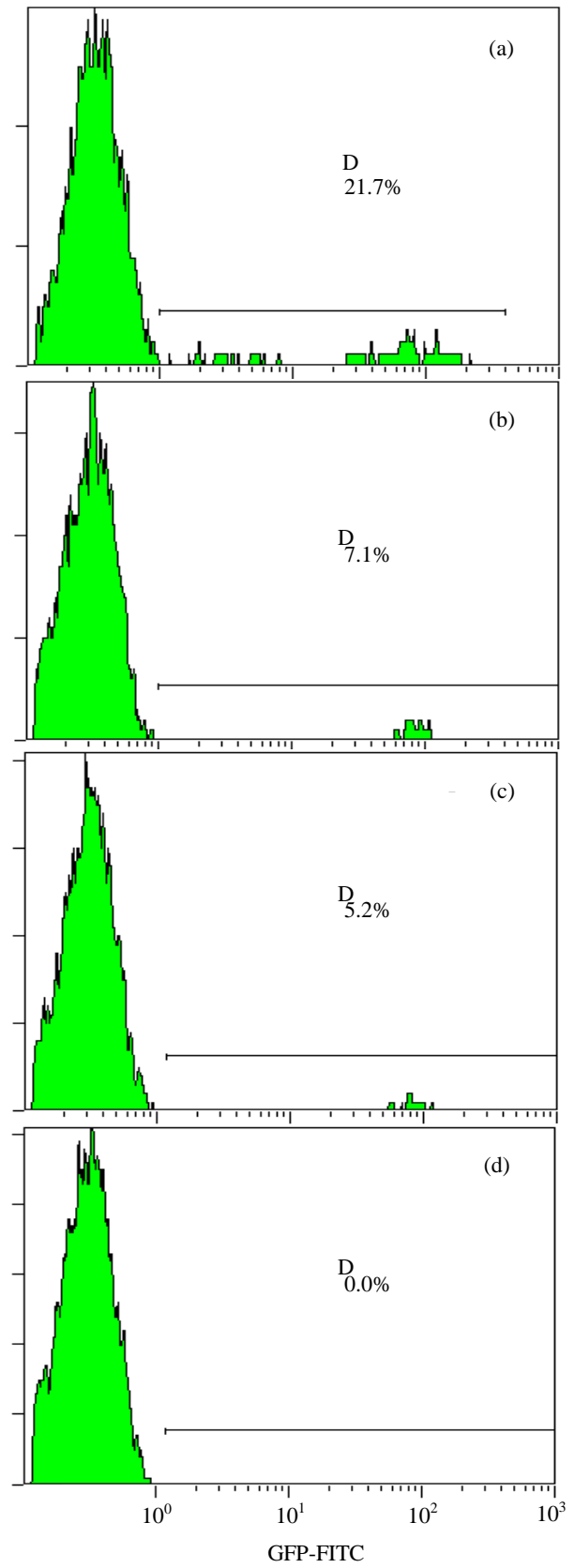

Fig. 1: At different days after transplantation, the percentage of GFP-positive cells in peripheral blood; a) About 18 days after transplantation, the percentage of GFP-positive cells in peripheral blood was $21.7 \%$ in one mice; b) About 39 days after transplantation, the percentage of GFPpositive cells in peripheral blood was $7.1 \%$ in one mice; c) About 53 days after transplantation, GFPpositive cells in peripheral blood was $5.2 \%$ in one mice; d) the percentage of GFP-positive cells in peripheral blood of mice was $0 \%$ after saline transfusion 

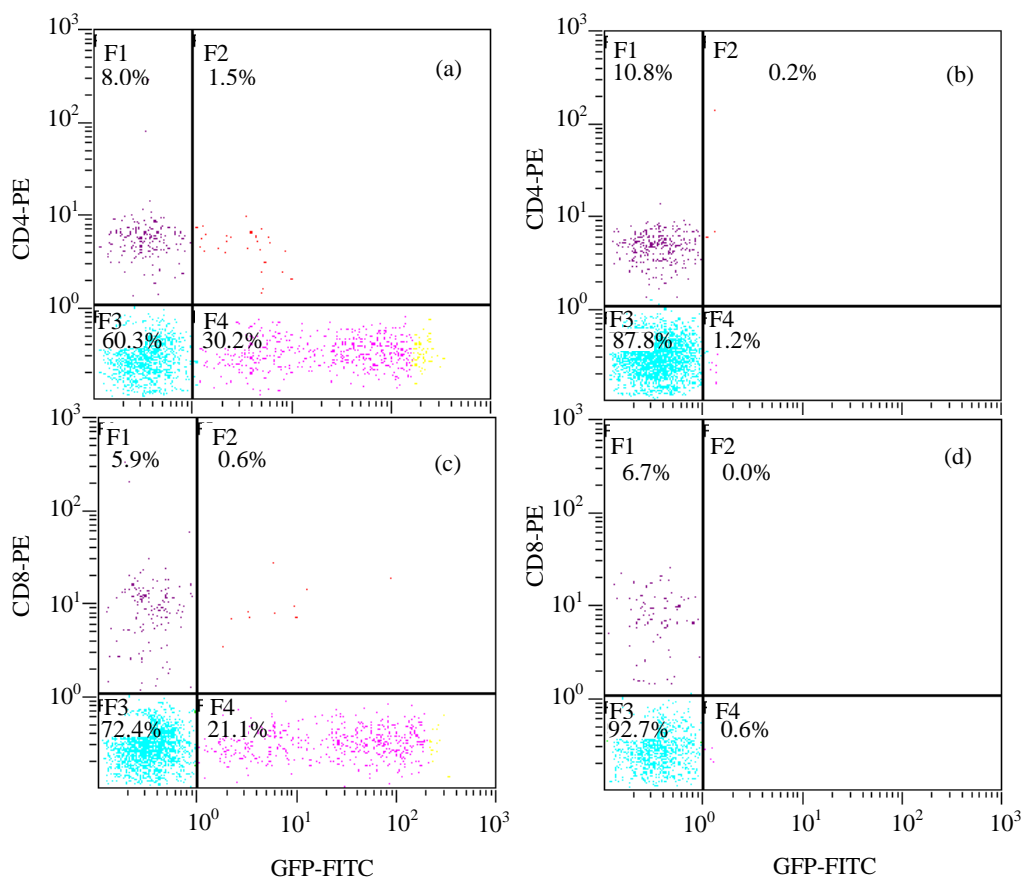

Fig. 2: About 18 days after transplantation, peripheral blood GFP and CD4-PE or CD8-PE double positive percentage; a) About 18 days after transplantation in $31.7 \%$ of the GFP-positive cells have $1.5 \%$ of CD4-PE positive cells; $b$ ) About 18 days after irradiation, the mice without infusion of liver cells CD4-PE and GFP double positive percentage was of $0.2 \%$; c) About 18 days after transplantation in $21.7 \%$ of the GFP-positive cells have $0.6 \%$ of CD8-PE positive cells; d) About 18 days after transplantation, the mice without infusion of liver cells GFP and CD8-PE double positive percentage was of $0 \%$

contain a certain amount of hematopoietic stem cells and play a hematopoietic reconstitution role or because the liver cells after transfusion to irradiated mice in vivo transdifferentiated into hematopoietic stem cells requires further detailed study. The research showed that after the mice infused the liver cells, their peripheral blood can be detected GFP and CD4-PE double-positive cells but also detected GFP and CD8-PE double-positive cells indicating that fluorescent mice liver cells survived in the female mice and converted into CD4+ and CD8 + T cells which are hematopoietic cell lineage markers (Ning et al., 2010).

That liver cell transfused to treat the half-lethally irradiated female and in the body converted into hematopoietic cells markers indicated that the liver cells had a new application. The cells contained hematopoietic stem cells or in vivo transdifferentiation into hematopoietic cells so that we will have a new proposal for the use of liver cells. The following researchers will continue to study the various components of liver cells and their roles for treating the hematopoietic function damaged mice. Further recommendations for the liver cells will be applied in aplastic anemia and other blood diseases in the future.
About 18 days after transplantation, GFP-positive cells in peripheral blood had a higher percentage of $26.55 \%$ and 39 and 53 days after transplantation, the percentage of GFP-positive cells was stable and reduced to 6.5 and $4.95 \%$. About 87 days after transplantation, the percentage of GFP-positive cells in peripheral blood was $1.7 \%$, the percentage of GFP-positive cells in bone marrow was of $1.4 \%$. Y chromosome positive rate in bone marrow was of $2 \%$. GFP-positive cells in bone marrow and the percentage of $\mathrm{Y}$ chromosome positive cells had no statistical significance compared GFP-positive cells in peripheral blood.

Therefore, the simplest way to detect hematopoietic reconstruction is about 90 days after transplantation to cut mice tails to collect blood to test the percentage of GFP-positive cells. The expensive fluorescence in situ hybridization method to detect Y-chromosome-positive rate positively correlated to the percentage of GFPpositive cells in peripheral blood. To collect peripheral blood is very easy, just cut tail to collect $<50 \mu \mathrm{L}$ blood, you can easily hemolyze to detect positive rate by flow cytometry. 
J. Anim.Vet. Adv., 11 (8): 1258-1264, 2012
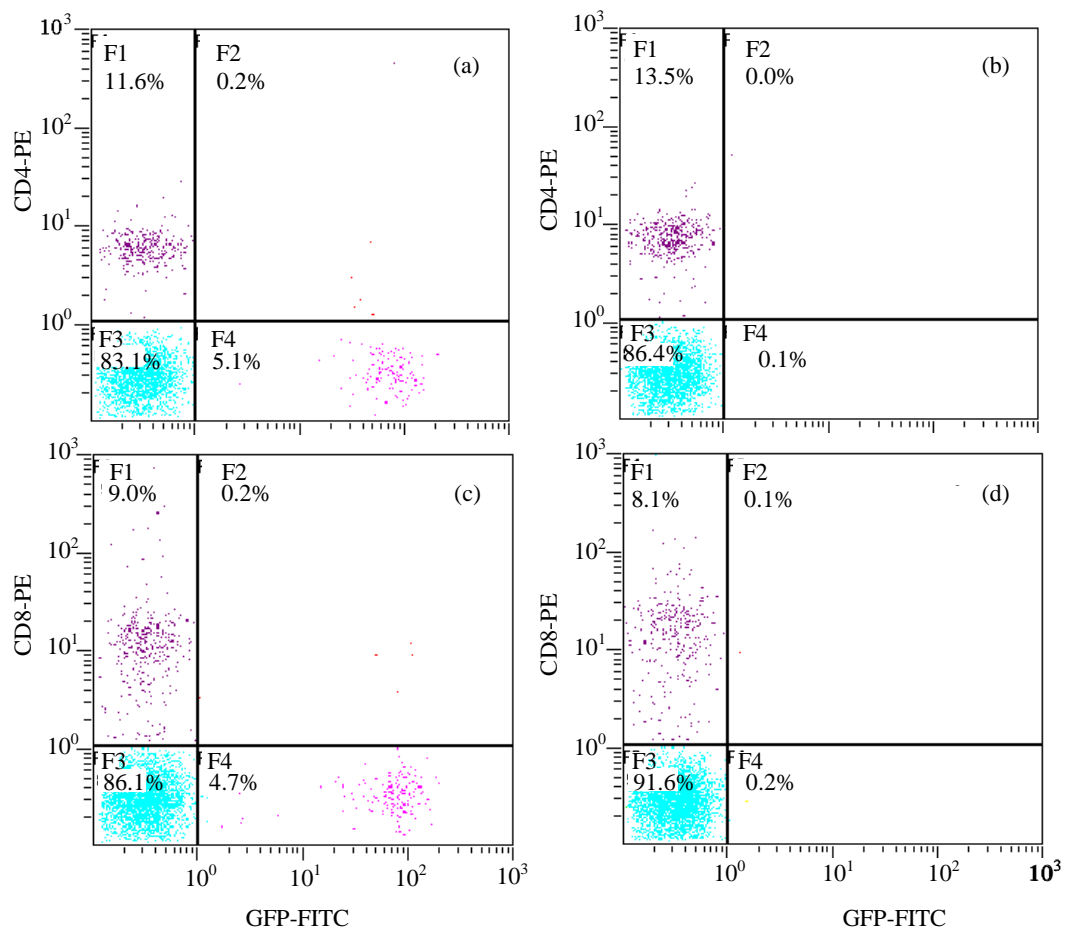

Fig. 3: About 53 days after transplantation, peripheral blood GFP and CD4-PE or CD8-PE double positive percentage. a) About 53 days after transplantation in $5.3 \%$ of GFP-positive cells had $0.2 \%$ of CD4-PE-positive cells; b) About 53 days after transplantation, the mice without infusion GFP and CD4-PE double positive percentage was of $0 \%$; c) About 53 days after transplantation in $4.9 \%$ of GFP-positive cells had $0.2 \%$ of the CD8-PE-positive cells; d) About 53 days after transplantation, the mice without infusion GFP and CD8-PE double positive percentage was of $0.1 \%$

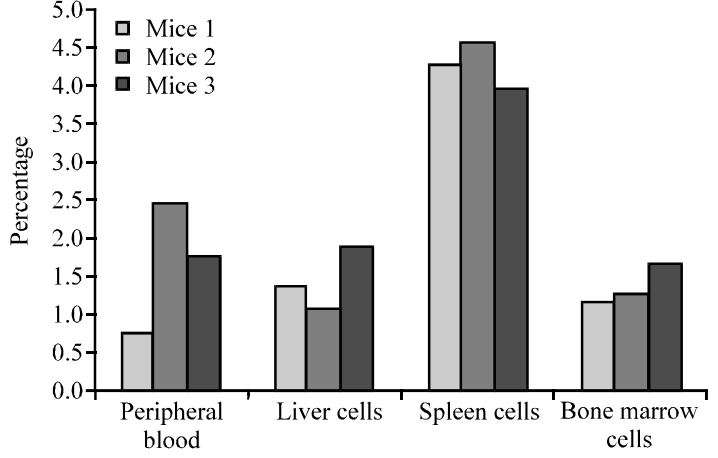

Fig. 4: About 87 days after transplantation, the percentage of GFP-positive cells in different cells of 3 mice. The results of pairwise comparison showed that the GFP cells positive rate in spleen cells was statistically significant higher than in other cells $(p=0.003)$

The mice were long-term survival and can be continuous monitored. It provides a simple method of identification of hematopoietic reconstitution. With simple and cheap way to be able to replace the expensive FISH test for the identification of hematopoietic reconstitution and reconstruction level provided a new idea about 87 days after transplantation, the three mice were sacrificed. The GFP-positive cells were detected in their liver, spleen and bone marrow, further evidencing that the transfused liver cell survived and played the reconstruction of hematopoietic function in female mice. The GFP-positive cells detected in spleen cells are higher than the percentage of GFP-positive cells in peripheral blood, liver and bone marrow. The comparison result was statistically significant $(\mathrm{p}=0.003)$.

The infused liver cells went through peripheral blood circulation and distributed in various organs of the female body and colonized in the spleen and played a role in hematopoietic reconstruction. The spleen is a hematopoietic organ, the survival of GFP-positive cells were most in the spleen. Compared to peripheral blood, liver and bone marrow cells implanted cell survival was statistically significant. The results suggested that adult mice liver cells had a certain amount of hematopoietic stem cells not so many as fetal liver cells but also survived in irradiated female body and played a 

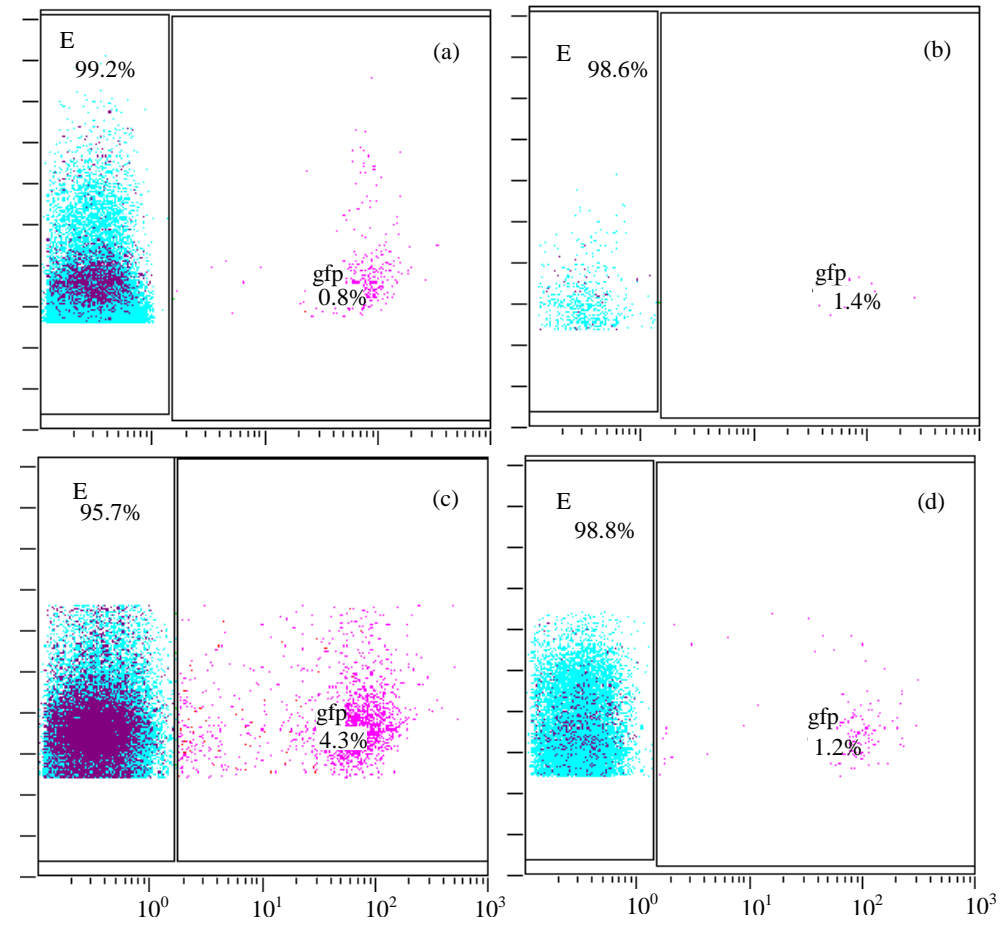

Fig. 5: About 87 days after transplantation, the flow cytometry results of GFP-positive rate in different cells; a) in peripheral blood; b) in liver cells; c) in spleen cells; d) in bone marrow cells

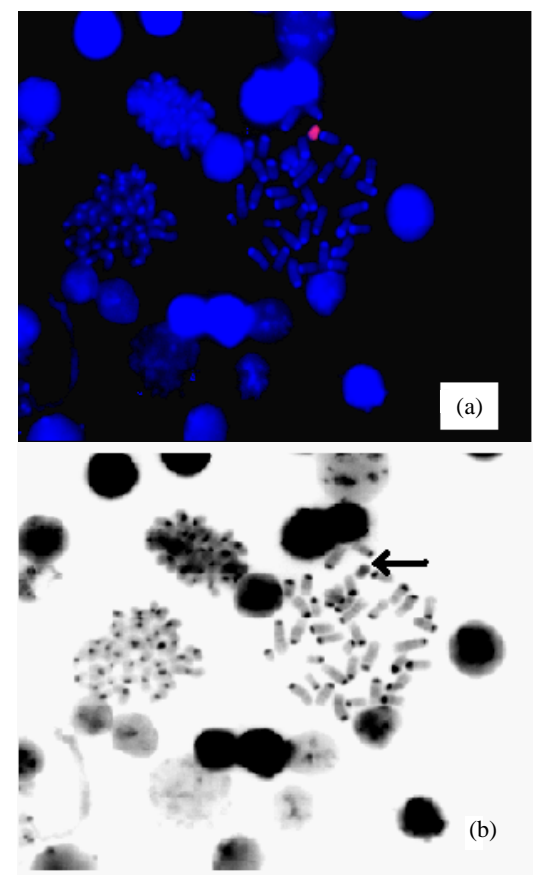

Fig. 6: Y chromosome was detected in bone marrow metaphase cleavage image by fluorescence in situ hybridization; a) red was Y chromosome; b) with a corresponding position of Fig. $6 \mathrm{a}$, the arrow was $\mathrm{Y}$ chromosome without a centromere role. If not because of adult mice liver cells with hematopoietic stem cells, another hypothesis was liver cell transdifferentiated into hematopoietic stem cells in female mice. Specific mechanisms need to be studied in the future.

In recent years, many studies used liver cell transplantation for treatment of liver failure and metabolic diseases, treatment have been successful in animal models and human (Parveen et al., 2011; Rao et al., 2008; Smets et al., 2008). This method is simpler than liver transplantation. It is technically simple and less expensive, cells from a single donor can be used for many recipients and they can be cryopreserved and are less immunogenic than solid organs (Stephenne et al., 2006, $2005,2007)$. We used liver cells to irradiated mice by intravenous infusion and observed the distribution and evolution of liver cells in vivo. It offers the experimental data for liver cell transplantation to refer.

\section{CONCLUSION}

The studies have shown that liver cells have the function of hematopoietic reconstruction which provides a new stratege for aplastic anemia and other blood system diseases. It is still unclear because of liver cells themselves containing hematopoietic stem cells or 
because the liver cells in vivo transdifferentiated into hematopoietic stem cells, researchers need further study in the future.

\section{ACKNOWLEDGEMENTS}

This research was supported by National Natural Science Foundation of China (31172170) and China Postdoctoral Science Foundation special funding.

\section{REFERENCES}

Lysy, P.A., M. Najimi, X. Stephenne, A. Bourgois, F. Smets and E.M. Sokal, 2008. Liver cell transplantation for crigler-najjar syndrome type I: Update and perspectives. World J. Gastroenterol, 14: 3464-3470.

Meyburg, J., A.M. Das, F. Hoerster, M. Lindner and H. Kriegbaum et al, 2009b. One liver for four children: first clinical series of liver cell transplantation for severe neonatal urea cycle defects. Transplantation, 87: 636-641.

Meyburg, J., J. Schmidt and G.F. Hoffmann, 2009a. Liver cell transplantation in children. Clin. Transplant., 21: 75-82.

Najimi, M. and E. Sokal, 2004. Update on liver cell transplantation. J. Pediatr. Gastroenterol. Nutr., 39: 311-319.

Ning, L., E. Goossens, M. Geens, D. van Saen, I. van Riet, D. He and H. Tournaye, 2010. Mouse spermatogonial stem cells obtain morphologic and functional characteristics of hematopoietic cells In vivo. Hum. Reprod., 25: 3101-3109.
Nyamath, P., A. Alvi, A. Habeeb, S. Khosla, A.A. Khan and C.M. Habibullah, 2007. Characterization of hepatic progenitors from human fetal liver using CD34 as a hepatic progenitor marker. World J. Gastroenterol., 13: 2319-2323.

Pang, W., 2000. Role of muscle-derived cells in hematopoietic reconstitution of irradiated mice. Blood, 95: 1106-1108.

Parveen, N., A.K. Aleem, M.A. Habeeb and C.M. Habibullah, 2011. An update on hepatic stem cells: bench to bedside. Curr. Pharm. Biotechnol., 12: 226-230.

Rao, M.S., A.A. Khan, N. Parveen, M.A. Habeeb, C.M. Habibullah and G. Pande, 2008. Characterization of hepatic progenitors from human fetal liver during second trimester. World J. Gastroenterol., 14: $5730-5737$.

Smets, F., M. Najimi and E.M. Sokal, 2008. Cell transplantation in the treatment of liver diseases. Pediatr. Transplant., 12: 6-13.

Stephenne, X., M. Najimi, C. Sibille, M.C. Nassogne, F. Smets and E.M. Sokal, 2006. Sustained engraftment and tissue enzyme activity after liver cell transplantation for argininosuccinate lyase deficiency. Gastroenterology, 130: 1317-1323.

Stephenne, X., M. Najimi, F. Smets, R. Reding, J. de Ville de Goyet and E.M. Sokal, 2005. Cryopreserved liver cell transplantation controls ornithine transcarbamylase deficient patient while awaiting liver transplantation. Am. J. Transplant., 5: 2058-2061 .

Stephenne, X., O. Vosters, M. Najimi, C. Beuneu and K.N. Dung et al., 2007. Tissue factor-dependent procoagulant activity of isolated human hepatocytes: Relevance to liver cell transplantation. Liver Transpl., 13: 599-606. 